Tafurt Villarraga, D., Molina, A., \& Duque, G. (2021). Hábitos alimentarios de tres especies de peces lenguados (Pleuronectiformes: Achiridae) en la Bahía de Buenaventura, Pacífico Colombiano. Revista de Biología Tropical, 69(3), 1055-1068. https://doi.org/10.15517/rbt. v69i3.41922

https://doi.org/10.15517/rbt.v69i3.41922

\title{
Hábitos alimentarios de tres especies de peces lenguados (Pleuronectiformes: Achiridae) en la Bahía de Buenaventura, Pacífico Colombiano
}

\author{
Daniella Tafurt Villarraga ${ }^{1}$; (D) https://orcid.org/0000-0003-3723-7151 \\ Andrés Molina ${ }^{2}$; (D) https://orcid.org/0000-0003-1954-4393 \\ Guillermo Duque ${ }^{3}$; (D) https://orcid.org/0000-0002-2468-529X
}

1. Grupo de Investigación en Ecología y Contaminación Acuática. Universidad Nacional de Colombia, Sede Palmira, Carrera 32 Chapinero, vía Candelaria, Palmira, Colombia; dtafurtv@unal.edu.co

2. Grupo de investigación en Ecología y Contaminación Acuática, Instituto de Estudios en Ciencias del Mar CECIMAR, Atte. Invemar, Universidad Nacional de Colombia - Sede Caribe - Calle 25 \# 2-55, El Rodadero, Santa Marta, Colombia; aemolinas@unal.edu.co

3. Facultad de ingeniería y Administración. Universidad Nacional de Colombia, Sede Palmira, Carrera 32 Chapinero, vía Candelaria, Palmira, Colombia; gduquen@unal.edu.co

$$
\text { Recibido 21-V-2021. Corregido 22-VII-2021. Aceptado 09-IX-2021. }
$$

\section{ABSTRACT \\ Feeding habits of three species of sole fish (Pleuronectiformes: Achiridae) in Buenaventura Bay, Colombian Pacific}

Introduction: The dynamic of the environmental conditions influences the availability of the food resources for the estuarine organisms, affecting the food consumption of the benthic organism such as the soles, which are essential for establishing and monitoring the environmental quality of these ecosystems.

Objective: To determine the trophic habits of three species of the Achiridae family in Buenaventura Bay, Colombian Pacific.

Methods: Fish were captured using artisanal trawling. Then, the diet of each species was determined and different trophic indexes were calculated registering differences in alimentary habits among species.

Results: It was collected a total of 159 fishes of the genus Achirus, being the most abundant Achirus klunzingeri (98), then Achirus mazatlanus (44) and finally Achirus scutum (17). The diet of A. scutum was based on detritus, the diet of $A$. mazatlanus was mainly detritus and fish whereas the diet of $A$. klunzingeri presented a dynamic pattern based mainly on crustaceans and fish. The individuals of Achirus klunzingeri presented differences in the diet between the internal and external area of the estuary, in the internal zone the fish were the main prey while in the external zone were the crustaceans. The species $A$. klunzingeri and A. mazatlanus presented ingestion of microplastics, mainly elongated and colorless fibers which were present in individuals of all sizes. The dynamic of the trophic habits of $A$. klunzingeri was related to the environmental variables such as temperature, transparency and dissolved oxygen. For example, these three variables presented negative correlations with the item fishes, and positive for the crustacean category.

Conclusions: Despite the generalist nature of the studied species, the environmental conditions, not only influenced the food consumption dynamic, but also determined the presence of microplastics.

Key words: Achirus; trophic habits; microplastic fibers; Tropical Eastern Pacific; estuaries. 
Los estuarios son ecosistemas con una alta complejidad, habitado por especies altamente resistentes a variaciones en las condiciones ambientales (Day et al., 2012). Por otra parte, la descarga de los ríos introduce nutrientes, produciendo una alta productividad primaria en los estuarios, lo cual permite el flujo constante de energía y el desarrollo de organismos de diferentes niveles tróficos (Sandoval et al., 2014). Así mismo, los ecosistemas estuarinos constituyen un ambiente fundamental para numerosas comunidades humanas, al brindar diferentes servicios ecosistémicos, destacándose la pesca, de la cual dependen más de 1000 millones de personas como fuente de proteínas y recursos económicos (Peña \& Palacios, 2013) y el turismo, por la belleza paisajística de estos ecosistemas (Rodríguez \& Reul, 2010). Para conservar los procesos ecológicos que soportan los servicios ecosistémicos de los estuarios, es importante preservar la biodiversidad, debido a que es la base de la producción y flujo energético.

Los procesos ecológicos de los ambientes bentónicos en los estuarios son importantes para el flujo de alimentos y nutrientes entre la columna de agua y el sedimento, que a su vez sirve como sustrato y refugio (Bárbara \& Peña, 2006). Los organismos que habitan estos ambientes están adaptados de diferentes maneras para obtener alimentos; también hay un gran número de organismos que no habitan en el fondo pero que se alimentan de él, incluyendo a los peces (Day et al., 2012).

Los lenguados de la familia Achiridae (orden Pleuronectiformes), son peces planos que viven en los fondos marinos y estuarinos. En la costa Pacífica se encuentran 3 especies del género Achirus: A. klunzingeri, crece hasta $23 \mathrm{~cm}$ y habita en profundidades entre 10-40 $\mathrm{m} ;$ A. mazatlanus, alcanza un tamaño hasta de $23 \mathrm{~cm}$ y profundidades de 1-55 m; y A. scutum, alcanza un tamaño de $19 \mathrm{~cm}$ y habita en profundidades de 5-45 m (Froese \& Pauly, 2019; Robertson \& Allen, 2015). Las especies de la familia Achiridae son generalmente carnívoras, y basan su alimentación en crustáceos bentónicos, peces óseos, y poliquetos, sin embargo, se ha encontrado que la alimentación de los lenguados puede variar según la disponibilidad de alimentos, la cual podría estar influenciada por las condiciones ambientales, la actividad antrópica y el tipo de estuario (Duque et al., 2020; Norbis \& Galli, 2004).

El estudio de los hábitos alimentarios de la familia Achiridae permite conocer las interrelaciones con diferentes organismos estuarinos, y su variación podría representar cambios en el nicho ecológico dentro de la estructura de la comunidad (Guevara et al., 2007). El ser humano ha influenciado en la ecología trófica al introducir diferentes compuestos a los ecosistemas (Sousa et al., 2019), siendo los microplásticos (desechos plásticos inferiores a $5 \mathrm{~mm}$ ) de sumo interés por su cantidad, potencial de acumulación en los ecosistemas y por su ingesta accidental por animales marinos (Bermúdez-guzmán et al., 2020; Ory et al., 2018). Los microplásticos en los organismos pueden causar desnutrición al bloquear el tracto digestivo (Elías, 2015) e introducción de toxinas debido a que tienden a absorber y acumular contaminantes del agua circundante, como metales pesados y contaminantes orgánicos (Guo \& Wang, 2019). Estudios aseguran que una de las áreas de mayor concentración de microplásticos es la interfaz agua-sedimento y los $0.5 \mathrm{~cm}$ superiores de sedimentos; hábitats de los lenguados estudiados en el presente artículo (Martin et al., 2017).

Se han desarrollado estudios sobre los hábitos alimentarios de los lenguados Paralichthys orbignyanus, en el Océano Atlántico meridional: Rocha, Uruguay (Norbis \& Galli, 2004); Paralichthys patagonicus en costas argentino-uruguayas (Gonzalo, 2011); Syacium micrurum, en el atlántico oriental (Marques et al., 2009), además, en Argentina se realizaron estudios sobre las interacciones tróficas en zonas costera, incluyendo el lenguado Paralichthys isosceles (Pozzobon, 1987). Con estos estudios se puede concluir que los lenguados tienen una estrategia de alimentación generalista y relativamente diversificada, en donde las principales presas suelen ser moluscos, crustáceos, poliquetos y peces. 
En el Océano Pacífico, se evaluaron las interacciones tróficas de 5 especies de lenguados: Bothus leopardinus, Syacium ovale, Cyclopsetta panamensis, Cyclopsetta querna y Monolene assaedae, encontrando que los dos primeros basan su alimentación en crustáceos y los demás en peces y cefalópodos (Flores et al., 2013). En Colombia, se reportó la dieta de lenguados de la familia Paralichthyidae en el Pacífico, compuesta principalmente por peces óseos y crustáceos bénticos (López \& Arcila, 2002). Sin embargo, la ecología trófica de la familia Achiridae ha sido poco estudiada. En el estuario Bahía de Buenaventura, se ha encontrado que $A$. klunzingeri y A. mazatlanus son especies residentes del estuario, presentando alta densidad, biomasa y frecuencia de ocurrencia, mientras $A$. scutum se ha registrado como la especie menos abundante de la familia Achiridae en este estuario (Molina et al., 2020).

El estudio de los hábitos alimentarios de la familia Achiridae, permite aportar al entendimiento de las relaciones tróficas en el estuario, que actualmente están siendo impactadas por actividades antrópicas y de las cuales se desconoce su influencia en peces de esta familia en el Pacífico colombiano, además de conocer la dinámica del flujo de energía desde el bentos hacia la columna de agua y cómo estos procesos son influenciados por variaciones ambientales. En este sentido, el objetivo de esta investigación fue determinar la influencia de las variaciones de las condiciones ambientales en la ecología trófica de tres especies de lenguados de la familia Achiridae; Achirus scutum, A. mazatlanus y A. klunzingeri, en la Bahía de Buenaventura, Pacífico colombiano. Adicionalmente, se evaluó el consumo de microplásticos en estas especies, para generar una línea base de información.

\section{MATERIALES Y MÉTODOS}

El estuario Bahía de Buenaventura, localizado en el Pacífico colombiano (353'47' $\mathrm{N} \& 77^{\circ} 04^{\prime} 40^{\prime}$ 'W), es el principal puerto de Colombia (Pérez, 2007). La precipitación es bimodal, presentando sus mayores valores entre septiembre y octubre y los más bajos entre febrero y marzo (Otero et al., 2007), con precipitación promedio anual de $7400 \mathrm{~mm}$. Así mismo, la temperatura promedio es 25.9 ${ }^{\circ} \mathrm{C}$ (Enriquez et al., 2014). Actualmente cuenta con 423927 habitantes, además de tener una vocación turística con 243915 visitas anuales según la Autoridad Marítima Nacional (Gallego Perez \& Selvaraj, 2019; Lusher et al., 2013).

La Bahía de Buenaventura presenta una profundidad media de $5 \mathrm{~m}$, y la desembocadura de dos ríos principales, ríos Anchicayá y Dagua, que le dan características de un estuario positivo (Cantera \& Blanco, 2001). Esta Bahía presenta una fuerte intervención antrópica, afectada principalmente por la mala disposición de las 4000 toneladas de residuos mensuales que son producidos en la ciudad (Riascos et al., 2019), además del arrastre y posterior descarga de contaminantes de los ríos efluentes. Estas condiciones hacen que, entre la basura marina del estuario, los microplásticos estén presentes con promedios de densidad de $359.6 \pm 88.0$ partículas $/ \mathrm{kg}$ en sedimentos para el 2019, siendo un contaminante importante en la bahía (Vásquez et al., 2021).

El estuario fue dividido en dos zonas de muestreo: estuario interno (EI) y estuario externo (EE), según el gradiente de salinidad, geomorfología y las características ambientales; monitoreando dos estaciones en cada zona cada campaña de muestreo (Duque \& Cogua, 2016). En el EI hay mayor influencia de los ríos $\mathrm{y}$ en el EE se presenta una mayor influencia marina (Fig. 1).

Métodos de muestreo: Las variables fisicoquímicas fueron medidas in situ tres veces, una vez antes de cada arrastre para muestras de peces, a una profundidad de $50 \mathrm{~cm}$. Las variables medidas fueron: salinidad (psu), temperatura $\left({ }^{\circ} \mathrm{C}\right)$, oxígeno disuelto $\left(\mathrm{mg} \mathrm{l}^{-1}\right)$ y $\mathrm{pH}$, con una sonda multiparametrica (Thermo Scientific Orion Five Stars) y transparencia $(\mathrm{cm})$ con un disco secchi.

Se recolectaron 159 individuos de 3 especies de la familia Achiridae; 17 de Achirus scutum, 44 de Achirus mazatlanus y 98 de 


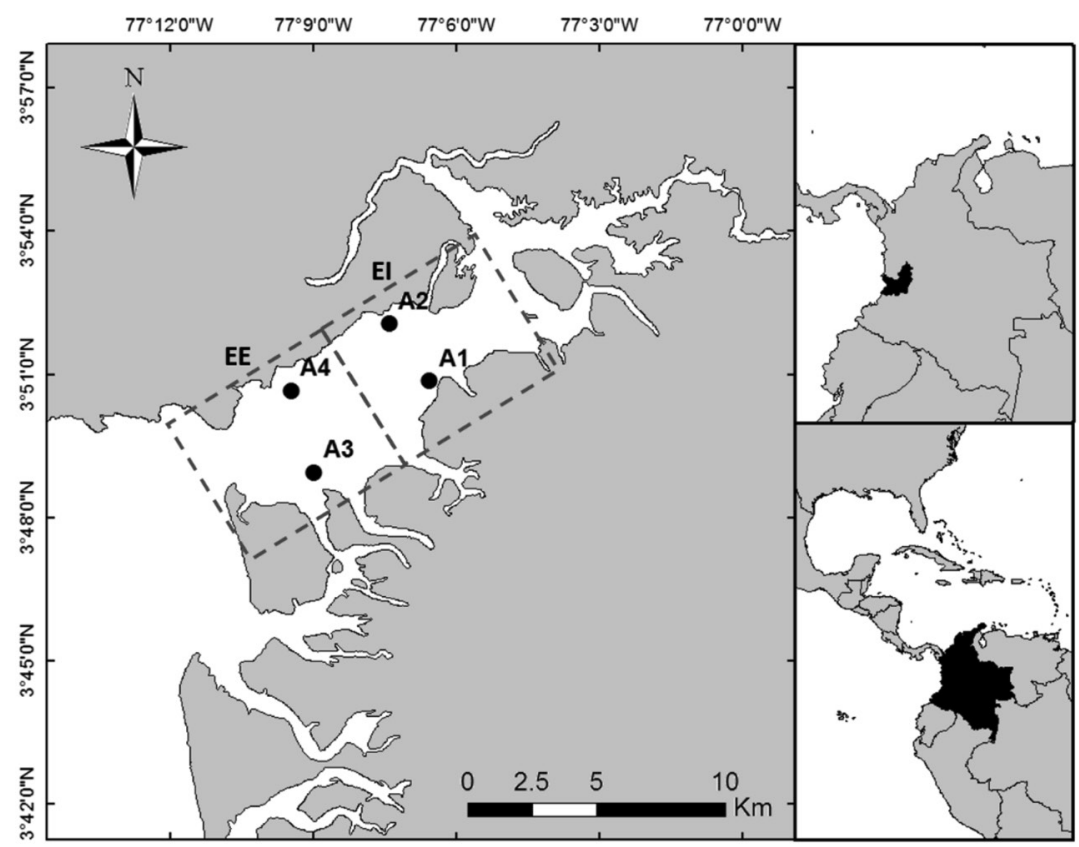

Fig. 1. Bahía de Buenaventura: área de estudio y estaciones de muestreo. Las líneas punteadas representan las dos zonas de muestreo, Estuario Interno (EI) y Estuario Externo (EE). Los puntos representan los sitios de muestreo.

Fig. 1. Buenaventura Bay: study area and sampling stations. The dotted lines represent the two sampling zones, Internal Estuary (EI) and External Estuary (EE). The dots represent the sampling sites.

Achirus klunzinger, en 9 muestreos realizados en la época seca, primer semestre, y en la época lluviosa, segundo semestre (Molina et al., 2020), del 2015 al 2017. Las muestras se recolectaron usando pesca de arrastre artesanal, haciendo tres arrastres de 10 minutos. $\mathrm{La}$ red tenía un ojo de malla de $25.4 \mathrm{~mm}$, relinga inferior de $3.6 \mathrm{~m}$ y relinga superior de $3.1 \mathrm{~m}$. Por los tamaños de la malla, se asegura que es muy poco probable que el microplástico ingerido por los peces se pueda deber a microplástico atrapado por la malla y posteriormente ingerido en la misma (Lusher et al., 2013, 2017). Los arrastres se realizaron a velocidades entre 3.1 y $4.0 \mathrm{~km} \mathrm{~h}^{-1}$, arrastrando áreas entre 790 y $1011 \mathrm{~m}^{-2}$.

Fase laboratorio: Los ejemplares recolectados fueron identificados siguiendo diferentes claves de identificación (Fischer et al., 1995; Froese \& Pauly, 2019; Nelson, 2006; Robertson \& Allen, 2015), midiendo la longitud total
(LT [cm]), longitud estándar (LS [cm]), y peso húmedo $(\mathrm{g})$. Así mismo, se extrajo el estómago, conservándolo en formol al $4 \%$. Los componentes alimentarios fueron observados en el estereoscopio a $3 \mathrm{X}$ y separados en categorías, almacenados individualmente en recipientes y posteriormente congelados para evitar la contaminación (Dehaut et al., 2019). Además, se contó el número de ítem por individuo, se secaron a $70{ }^{\circ} \mathrm{C}$, por 24 horas y se determinó el peso seco en una balanza analítica (Majdi et al., 2018). A los microplásticos hallados se le realizaron pruebas de elasticidad y dureza para verificar que no correspondiera a estructuras celulares u orgánicas, además de evaluación visual detallada de la forma, color y textura (Lusher et al., 2017).

Se tuvieron en cuenta algunas recomendaciones con el fin de minimizar la contaminación cruzada, por esto, se realizó un tipo de muestreo que no tiene contacto con el sistema digestivo (McGoran et al., 2017); además, el sitio de 
procesamiento de muestras fue un laboratorio cerrado, con circulación del aire controlada y la mesa de trabajo fue limpiada previamente con alcohol. Se utilizó bata de algodón, guantes de un solo uso y elementos metálicos y de vidrio que antes de cada uso fueron limpiados y revisados bajo el estereoscopio para detectar contaminación (Lusher et al., 2013).

Análisis de datos: Para el análisis de datos no se tuvo en cuenta el material digerido, con los demás ítems se calculó la Composición Numérica (\% CN) (Ec. 1), Composición
Gravimétrica (\% CG) (Ec. 2), y Frecuencia de Ocurrencia de presas (\% FO) (Ec. 3), clasificándolas en: constantes (presente en más de $50 \%$ de las muestras), accesoria (entre 25 y $50 \%$ ) y accidental (menos de $25 \%$ ) (Alessandro et al., 2020; Duque \& Acero, 2003b). La importancia de cada presa fue determinada por el Índice de Importancia Relativa (IIR) (Ec. 4) clasificando en grupos tróficos de importancia baja (0-10\%), secundaria (10-40\%) y alta (40$100 \%$ ). Así mismo, se determinó el coeficiente de vacuidad (CV) (Ec. 5) (Olaya \& Arellano, 2012; Cogua et al., 2013).

$$
\begin{gathered}
\% C N=100 * \frac{\text { Número de presas del item } i}{N^{o} \text { total de presas }}(\text { Ec. } 1) \\
\% C G=100 * \frac{\text { Número de presas del item } i}{\text { Peso de todas las presas }}(\text { Ec. } 2) \\
\% F O=100 * \frac{\text { Ocurrencias de presas del item } i}{N^{o} \text { total de estómagos con alimento }}(\text { Ec. } 3) \\
\% \text { IIR }=F O * C G(\text { Ec. } 4) \\
C V=100 * \frac{N^{o} \text { de estómagos vacios }}{N^{o} \text { total de estómagos analizados }}(\text { Ec. } 5)
\end{gathered}
$$

Para identificar la dinámica espacio-temporal de las variables ambientales y tróficas se realizó un Escalamiento Multidimensional No Métrico (nMDS), usando matrices de similitud calculadas con el índice de Bray-Curtis y datos sin transformar (Clarke et al., 2014); así mismo, para identificar diferencias significativas se realizaron Análisis de Varianza Multivariado Permutacional (PERMANOVA), utilizando 9999 permutaciones y $\alpha<0.05$ como diferencia estadísticamente significativa (Clarke et al., 2014; Marti, 2017). Además, se realizaron análisis de correlación por rango de Spearman, para medir la asociación entre variables ambientales y alimentación (Martinez et al., 2009; Martinez et al., 2019).

\section{RESULTADOS}

Descripción ambiental: Se presentaron variaciones significativas de las condiciones ambientales según la época del año (lluviaseca) y la zona (interna-externa) en la salinidad y el $\mathrm{pH}$. En general, la salinidad tuvo valores entre 11.6 y 30 psu, presentando diferencias significativas entre épocas de muestreo $(\mathrm{p}($ Perm $)=0.0005)$. Los valores más altos se registraron en época seca - EE $(25.92 \pm 3,28)$, y los más bajos en época de lluvia - EI (15.87 \pm 3.47). Por otra parte, el pH varió desde 7 hasta 8.8 unidades, presentó diferencias significativas en la interacción épocas-zonas $(\mathrm{p}($ Perm $)=$ $0.0342)$. Los valores más altos se registraron en época seca - EE $(7.99 \pm 0.27)$, y los más bajos en época seca - EI $(7.63 \pm 0.30)$.

El oxígeno disuelto (OD) presentó valores

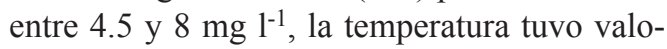
res entre 25 y $32{ }^{\circ} \mathrm{C}$, y la transparencia en la columna de agua osciló de 16 a $128 \mathrm{~cm}$. No se determinaron diferencias significativas en las variables analizadas para estas condiciones ambientales (Tabla 1). 
TABLA 1

Promedio de las variables ambientales en temporada seca y lluviosa, dividido en estuario interno y externo con el error estándar

TABLE 1

Average of the environmental variables in the dry and rainy season, divided into internal and external estuaries with standard error

\begin{tabular}{lcccccccc}
\hline \multirow{2}{*}{ Variables ambientales } & \multicolumn{4}{c}{ Seco } & \multicolumn{4}{c}{ Lluvia } \\
\cline { 2 - 10 } & Interno & \pm & Externo & \pm & Interno & \pm & Externo & \pm \\
\hline Temperatura $\left({ }^{\circ} \mathrm{C}\right)$ & 28.69 & 0.78 & 28.88 & 1.30 & 28.48 & 0.38 & 28.36 & 1.46 \\
Transparencia $(\mathrm{cm})$ & 67.87 & 30.83 & 65.42 & 18.10 & 44.94 & 14.38 & 51.67 & 14.02 \\
$\mathrm{pH}$ & 7.63 & 0.30 & 7.99 & 0.27 & 7.78 & 0.10 & 7.73 & 0.28 \\
Salinidad $(\mathrm{psu})$ & 23.47 & 4.31 & 25.92 & 3.28 & 15.87 & 3.47 & 19.42 & 4.60 \\
Oxígeno Disuelto $(\mathrm{mg} / \mathrm{l})$ & 5.45 & 0.51 & 6.06 & 0.56 & 6.21 & 1.28 & 6.53 & 1.15 \\
\hline
\end{tabular}
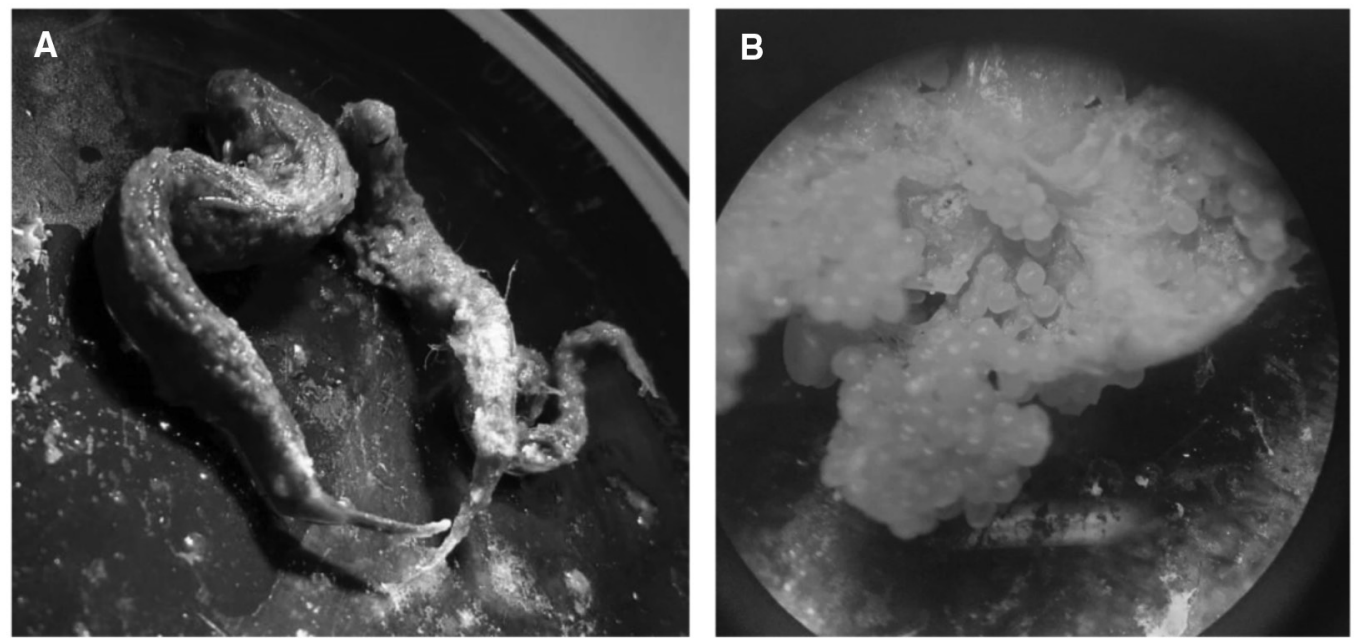

Fig. 2. Contenido estomacal familia Achiridae. A. Peces. B. Huevos, ingeridos por las especies A. mazatlanus y A. klunzingeri.

Fig. 2. Stomach content family Achiridae. A. Fish, B. Eggs, ingested by the species A. mazatlanus and A. klunzingeri.

Descripción trófica: Se encontraron 14 tipos de ítems en los estómagos de las 3 especies de la familia Achiridae que fueron agrupados en cinco categorías: 1) Peces, lográndose identificar individuos de Syngnathus sp. (Fig. 2A), 2) Crustáceos, agrupando cangrejos, camarones, copépodos e isópodos; 3) Detritos, incluyendo el material vegetal; 4) Material digerido; y la última categoría 5) Otros, incluye anélidos, nemátodos, huevos (Fig. 2B), restos de invertebrados y fibras plásticas.

El análisis estadístico permitió encontrar diferencias significativas entre especies en cuanto a la alimentación, siendo $A$. klunzingeri diferente de $A$. mazatlanus $(\mathrm{p}($ Perm $)=0.0002)$ y $A$. scutum $(\mathrm{p}($ Perm $)=0.0005)$; por el contrario, A. mazatlanus y A. scutum no presenta diferencias significativas en la alimentación. Además, se observó mayor presencia de detritus en A. mazatlanus y A. scutum, mientras A. klunzingeri se alimentó principalmente de crustáceos y peces.

Para $A$. scutum $(\mathrm{N}=17)$ la LT promedio fue $12.0 \pm 0.8 \mathrm{~cm}$, con un $\mathrm{CV}$ de $0 \%$, identificando los detritos como alimento constante $(69.5 \%$ FO) y como el ítem principal (65.1\% IIR). 
TABLA 2

Composición de la dieta de A. scutum, A. mazatlanus y A. klunzingeri

TABLE 2

Composition of the diet of A. scutum, A. mazatlanus y A. klunzingeri

\begin{tabular}{|c|c|c|c|c|c|c|c|c|c|c|c|c|}
\hline \multirow{2}{*}{ Categorías } & \multicolumn{4}{|c|}{ A. scutum } & \multicolumn{4}{|c|}{ A. mazatlanus } & \multicolumn{4}{|c|}{ A. klunzingeri } \\
\hline & $\% \mathrm{CG}$ & $\% \mathrm{CN}$ & $\% \mathrm{FO}$ & IIR & $\% \mathrm{CG}$ & $\% \mathrm{CN}$ & $\% \mathrm{FO}$ & IIR & $\% \mathrm{CG}$ & $\% \mathrm{CN}$ & $\% \mathrm{FO}$ & IIR \\
\hline Peces & 1.7 & 7.4 & 4.3 & 0.1 & 60.4 & 14.3 & 14.8 & 89.5 & 59.9 & 15.2 & 29.9 & 17.9 \\
\hline Crustáceos & 3.1 & 11.1 & 13 & 0.4 & 17.6 & 21.4 & 18.5 & 32.7 & 36.5 & 24.6 & 32.8 & 12 \\
\hline Detritos & 93.6 & 59.3 & 69.6 & 65.1 & 19.8 & 46.4 & 48.1 & 95.3 & 2.25 & 6.64 & 20.9 & 0.47 \\
\hline Otros & 1.5 & 22.2 & 13 & 0.2 & 2.16 & 17.9 & 18.5 & 3.99 & 1.35 & 53.6 & 16.4 & 0.22 \\
\hline
\end{tabular}

\% CG: Porcentaje en peso; \% CN: Porcentaje en número; \% FO: Porcentaje de frecuencia; IIR: Índice de importancia relativa de los ítems alimentarios de los estómagos de las tres especies en estudio.

\% CG: Weight percentage; \% CN: Percentage in number; \% FO: Frequency of ocurrence; IIR: Relative Importance Index of the stomach content items of the three species under study.

Además, se determinaron como presas accidentales los crustáceos (13\% FO), los peces (4.3 $\%$ FO) y otros (13.04 \% FO), compuestos por nematodos y restos de invertebrados (Tabla 2). Esta especie no presentó diferencias significativas en la alimentación entre épocas, zonas, ni en la interacción época-zona.

Por otra parte, A. mazatlanus $(\mathrm{N}=44)$ presento LT promedio de $13.3 \pm 1.9 \mathrm{~cm}$, con CV de $29.5 \%$, donde las presas principales fueron detritos y peces (IIR: 95.3 y $89.5 \%$, respectivamente), siendo los detritus un ítem accesorio (48.1\% FO) y los peces presa accidental (14.8 $\%$ FO). Así mismo, se identificaron como presas accidentales los crustáceos (18.5 \% FO) y otros (18.5 \% FO), compuestos por huevos, anélidos y fibra plástica (Tabla 2). Esta especie no presentó diferencias significativas en la alimentación entre épocas, zonas, ni en la interacción época-zona.

Finalmente, para A. klunzingeri $(\mathrm{N}=98)$ se encontró un CV de $28.5 \%$, con LT promedio de $16.1 \pm 3.7 \mathrm{~cm}$. En general, la alimentación se basó en crustáceos (32.8 \% FO, $12 \%$ IIR) y peces (29.8 \% FO, $17.9 \%$ IIR), seguidas por los detritos (20.9 \% FO), y otros (16.4\% FO) compuesto por isópodos, copépodos, fibra plástica, huevos y restos de invertebrados (Tabla 2). Se encontraron diferencias significativas entre zonas para \% FO $(\mathrm{p}($ Perm $)=0.0019)$ y \% IIR $(p($ Perm $)=0.0011)$. En el EI los peces fueron la presa más importante, clasificada como constante (50\% FO y $9.3 \%$ IIR), y los crustáceos $(21.4 \% \mathrm{FO})$, los detritos (17.9\% FO), y otros (10.7\% FO), compuesto por huevos y fibra plástica, como ítems accidentales. Por el contrario, en el EE los crustáceos fueron la presa más importante, clasificada como constante $(56.3 \%$ FO) y los peces, detritos y otros, compuesto por restos de invertebrados y fibra plástica, como ítems accidentales $(18.7 \% \mathrm{FO}$, $12.5 \%$ FO y $12.5 \%$ FO, respectivamente). Esta especie no presentó diferencias significativas en la alimentación entre épocas, ni en la interacción época-zona.

Ingesta de microplásticos: En los estómagos de A. mazatlanus y A. klunzingeri se encontraron fibras plásticas sintéticas, alargadas e incoloras (Fig. 3A, Fig. 3B). En A. mazatlanus las fibras se clasificaron como ítem accidental, presente en el $6.12 \%$ de los organismos ( $\%$ FO), representando el $7.9 \%$ de los ítems (\% $\mathrm{CN})$ y fueron encontrados en época lluvia-EI y en época seca-EE, en individuos con LT entre 8.8 y $14.5 \mathrm{~cm}$ y peso entre 12 y $62 \mathrm{~g}$. Además, en $A$. klunzingeri las fibras se clasificaron como ítem accidental, presentes en el $3.8 \%$ de los organismos (\% FO), representando el $1.7 \%$ de los ítems $(\% \mathrm{CN})$ y fueron encontrados en todas las épocas y zonas, en individuos con LT de 17 a $29.5 \mathrm{~cm}$ y peso entre 89 y $578 \mathrm{~g}$. 

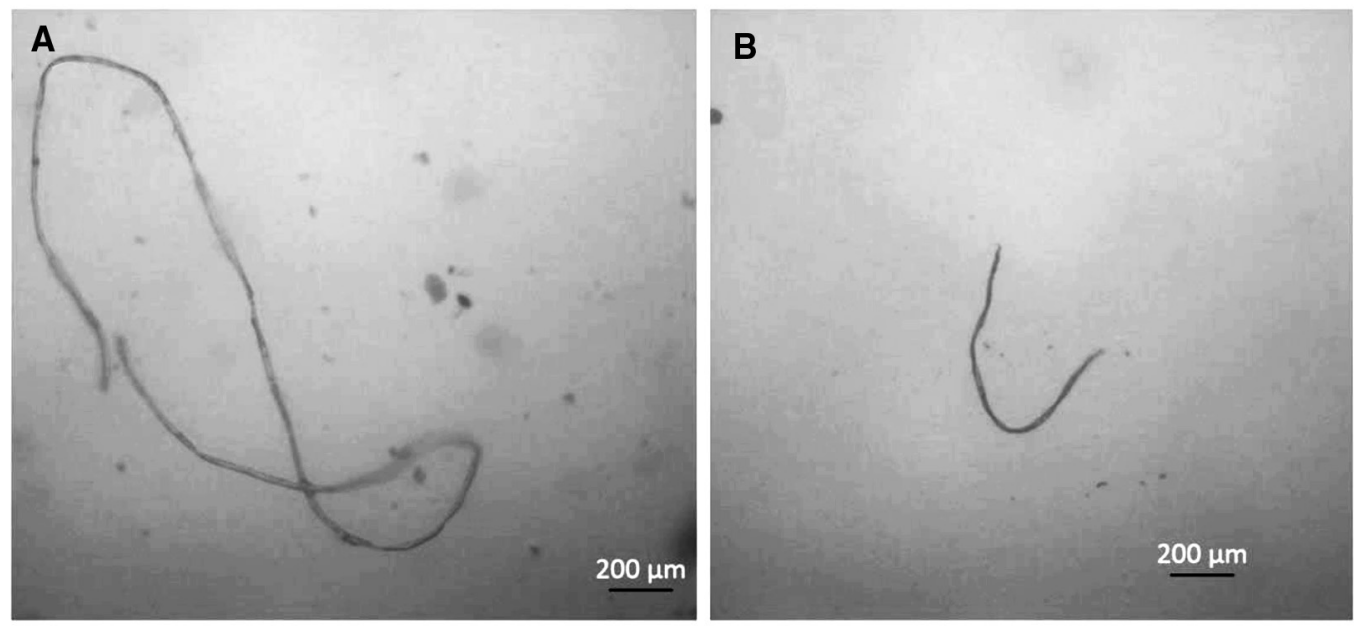

Fig. 3. Contenido estomacal familia Achiridae. A-B. Fibras plásticas ingeridas por las especies A. mazatlanus y A. klunzingeri.

Fig. 3. Stomach content family Achiridae. A- B. Plastic fibers ingested by the species A. mazatlanus and A. klunzingeri.

TABLA 3

Correlaciones por rango de Spearman entre las diferentes categorías alimentarias (IIR) y variables ambientales para $A$. klunzingeri

TABLE 3

Spearman rank correlations between different food categories (IIR) and environmental variables for A. klunzingeri

\begin{tabular}{lccccc}
\hline A. Klunzingeri & Salinidad & Oxígeno Disuelto $(\mathrm{mg} / \mathrm{l})$ & Temperatura $\left({ }^{\circ} \mathrm{C}\right)$ & Transparencia $(\mathrm{cm})$ & $\mathrm{pH}$ \\
\hline Peces & 0.0967 & $-0.3891^{* *}$ & $-0.5503 * * *$ & $-0.3863^{* *}$ & -0.2014 \\
Crustáceos & -0.0796 & $0.4084^{* *}$ & $0.3601 *$ & 0.1946 & 0.1434 \\
Detritos & -0.1627 & -0.0781 & 0.0507 & 0.1194 & -0.1126 \\
Otros & 0.1595 & 0.1491 & $0.3143^{*}$ & 0.118 & 0.2539 \\
\hline
\end{tabular}

$\mathrm{P}<0.05^{*} ; \mathrm{P}<0.01 * * ; \mathrm{P}<0.001 * * *$

Ecología trófica: Las dietas de A. scutum у A. mazatlanus no presentaron asociación significativa con las variables ambientales, mientras que el consumo de crustáceos y peces de $A$. klunzingeri se relacionó con la temperatura, el OD, y la transparencia. Se observó un aumento en el consumo de crustáceos cuando aumentó la temperatura y el OD. El consumo de peces fue mayor cuando disminuyó la temperatura, la transparencia y el OD. Así mismo, se encontró que el consumo de "otros", conformado por isópodos, copépodos, fibra plástica, huevos y restos de invertebrados, aumentó junto con la temperatura (Tabla 3 ).

\section{DISCUSIÓN}

Caracterización trófica: Las tres especies del género Achirus estudiadas basaron su alimentación en peces, crustáceos y detritos, con algunas preferencias de presas para cada especie; A. scutum se alimentó principalmente de detritos, A. mazatlanus de detritos y peces, y A. klunzingeri de crustáceos y peces. Las especies estudiadas pueden considerarse generalistas con preferencias de alimentación para cada especie, como es conocido para los lenguados, considerados predadores oportunistas-generalistas, al alimentarse según la 
disponibilidad y capacidad de captura (Esposito et al., 2010; J. Flores et al., 2013; Gonzalo, 2011; Marques et al., 2009; Norbis \& Galli, 2004). Esto concuerda con lo encontrado en el Pacífico central mexicano donde reportan a cinco especies de peces del orden Pleuronectiformes como consumidoras de peces y crustáceos; Monolene assaedae, Cyclopsetta panamensis y C. querna, consumen más peces que crustáceos y Bothus leopardinus y Syacium ovale prefieren los crustáceos sobre los peces (Flores et al., 2013).

Las diferencias en la alimentación de $A$. klunzingeri entre las zonas del estuario posiblemente se deban a cambios en la oferta de presas. Para la Bahía de Buenaventura se ha registrado que en la parte externa hay mayor abundancia de macroinvertebrados que en la parte interna (Gamboa-García et al., 2018), lo cual podría explicar que los crustáceos fueran la presa más importante en EE. Por otra parte, se encontró una alta densidad de peces en la parte interna del estuario (Molina et al., 2020), lo cual podría explicar que los peces fueron la presa más importante para esta zona. Para algunos peces estuarinos la alimentación está relacionada con la abundancia y ocurrencia de las presas (Duque \& Acero, 2003a; Marques et al., 2009).

La alimentación varió según la talla promedio de cada especie. Existe una relación entre la talla de la especie y la competencia por alimentos, observándose en especies de tallas pequeñas una mayor competencia interespecífica (Grabowska et al., 2016; Munday et al., 2001), lo cual limita la posibilidad de adquirir una mayor variedad de presas, en cambio, en las especies de mayor talla se reduce la competencia, lo que permite escoger su alimentación, al punto de poder considerarse especialistas. En este estudio las especies con tallas grandes ( $A$. klunzingeri) logran alimentarse principalmente de crustáceos y peces, mientras que las tallas más pequeñas ( $A$. mazatlanus y $A$. scutum) se alimentan de detritus, el cual no es deseable energéticamente, por ser menos digeribles, representando un menor valor nutricional que otras presas alimentarias (Cervera et al., 2006).
De la misma manera, características propias de la especie también podrían explicar cambios en la dieta como: morfología, migraciones y estado de madurez (Flores et al., 2013; Flores et al., 2015), lo cual sugiere posibles estudios a futuro.

Ingesta de microplásticos: Se evidenció que individuos de un amplio rango de tallas de A. mazatlanus y A. klunzingeri ingirieron microplásticos, en todas las zonas y épocas de muestreo. Se ha reportado que estos microplásticos son ingeridos independientemente del tamaño de los peces (Wright et al., 2013), de manera similar con el presente estudio. Adicionalmente, se ha sugerido que el consumo de microplásticos es mayor en depredadores activos, al incorporar microplásticos presentes en sus presas (Wright et al., 2013).

La mayoría de estos microplásticos fueron fibras, cuya procedencia podría asociarse a textiles, artes de pesca mal dispuestos o a la inadecuada gestión de los residuos de poblaciones cercanas al estuario o a los ríos que desembocan en él. Estas fibras son consideradas microplásticos secundarios, al estar asociados a la descomposición por procesos físicos, químicos y biológicos de elementos más grandes (Sousa et al., 2019). Sin embargo, al ser un sistema abierto, es difícil conocer su procedencia (Elías, 2015), se necesita más investigación para comprender los mecanismos que influyen en el transporte, la deposición, la resuspensión y las interacciones posteriores de los microplásticos con la biota (Martin et al., 2017).

La mayor ingesta de fibras plásticas por $A$. mazatlanus puede estar asociada a su alto consumo de detritos, pues se ha reportado que las fibras plásticas representan la forma más abundante de microplásticos en los fondos marinos (Mu et al., 2019; Peng et al., 2017; Vásquez et al., 2021; C. Zhang et al., 2019; D. Zhang et al., 2020). Para los sedimentos de Buenaventura, se han reportado las fibras plásticas como uno de los microplásticos más abundantes, presentándose las mayores abundancias en el estuario interno $(302.2 \pm 84.5$ partículas $/ \mathrm{kg})$ 
(Vásquez et al., 2021), lo que podría explicar su consumo incidental.

La especie $A$. scutum no presentó ingesta de microplástico, esto puede deberse al tamaño de la muestra, la cual está directamente relacionada con la abundancia del pez en el estuario. Es difícil determinar si esta ausencia de microplásticos se debe a una característica propia de la especie o específicamente corresponde al tamaño de la muestra; es necesario evaluar esta posibilidad en estudios futuros con un tamaño de muestra mayor.

Condiciones ambientales y ecología trófica: Según el análisis de la ecología trófica, las variables ambientales que influyeron en la alimentación de A. klunzingeri fueron: temperatura, OD y transparencia. Algunos factores como la latitud, hora del día e impactos antrópicos determinan la temperatura del agua (Du et al., 2021), influyendo también en la ecología trófica de los peces (Duque \& Acero, 2006; Galvis et al., 2005). En este estudio, $A$. klunzingeri, presentó mayor consumo de peces cuando disminuyó la temperatura, el oxígeno disuelto y la transparencia, características del estuario interno. Así mismo, en cuatro estuarios del Pacífico mexicano central, se evidenció que la disminución de la temperatura y la transparencia aumentó la abundancia de peces consumidores primarios (Sandoval et al., 2014). De acuerdo a lo anterior, el aumento en el consumo de peces en EI se asocia a la disponibilidad de presas bajo las condiciones del hábitat marino (Chango \& Nacimba, 2015). En contraste, el consumo de crustáceos se relacionó con aumentos de temperatura y OD, condiciones ambientales típicas del estuario externo, favorables para la mayoría de organismos estuarinos tropicales. Por esto, el mayor consumo de crustáceos en esta zona posiblemente se deba a que son más abundantes en condiciones con influencia marina en la Bahía de Buenaventura (Gamboa-García et al., 2018).

En el estuario las variables ambientales OD, temperatura y transparencia no se ven fuertemente afectadas por la influencia de los ríos ni por el tiempo atmosférico. Por el contrario, y concordando con la literatura, en épocas de lluvia y estuario interno, la salinidad y el $\mathrm{pH}$ disminuyen significativamente. El EE tiene mayor influencia marina, por el contrario, el EI está influenciado fuertemente por los ríos Dagua y Anchicayá (L. Otero, 2005) que, según estudios, la descarga de agua dulce representa un factor importante en la distribución y abundancia de los organismos estuarinos (França et al., 2011; Pichler et al., 2015), además la riqueza de las especies presenta correlaciones negativas con la salinidad (Sosa-López et al., 2007). Por otro lado, la época de lluvia representa condiciones más óptimas para la supervivencia de los peces debido al aumento de productividad primaria y secundaria (Pichler et al., 2015). Por lo anterior, se evidencia que el cambio en algunas condiciones ambientales del estuario está relacionado directamente con la época y la zona del estuario, las cuales además determinan la disponibilidad de alimentos para los organismos estudiados.

Los hallazgos aquí documentados permiten expandir los conocimientos previos acerca de los peces de la familia Achiridae, determinarlo como un organismo predador oportunista según la disponibilidad de su hábitat, pero con algunas preferencias de alimentación, además sirven como base para investigar la presencia e ingesta de microplásticos en organismos estuarinos en la Bahía de Buenaventura.

Declaración de ética: los autores declaran que todos están de acuerdo con esta publicación y que han hecho aportes que justifican su autoría; que no hay conflicto de interés de ningún tipo; y que han cumplido con todos los requisitos y procedimientos éticos y legales pertinentes. Todas las fuentes de financiamiento se detallan plena y claramente en la sección de agradecimientos. El respectivo documento legal firmado se encuentra en los archivos de la revista.

\section{AGRADECIMIENTOS}

Los autores agradecen a la Universidad Nacional de Colombia, por el apoyo 
institucional y económico mediante el proyecto "Efectos de operaciones de dragado en la comunidad de organismos estuarinos de la Bahía de Buenaventura, Pacifico colombiano" código Hermes 34779. Así mismo, agradecemos al grupo de investigación en Ecología y Contaminación Acuática por el apoyo en el campo y laboratorio.

\section{RESUMEN}

Introducción: La dinámica de las condiciones ambientales influencia la variación del alimento disponible para los organismos estuarinos, afectando el consumo de alimento de los organismos bentónicos como los lenguados, esenciales para establecer y monitorear la calidad ambiental en estos ecosistemas.

Objetivo: Determinar los hábitos tróficos de tres especies de la familia Achiridae en la Bahía de Buenaventura, Pacífico colombiano.

Métodos: Los peces se recolectaron por medio de pesca de arrastre artesanal. Así mismo, en laboratorio se determinó la dieta de cada una de las especies y se calcularon diferentes índices tróficos, registrando diferencias en los hábitos alimentarios entre estas especies.

Resultados: Se recolectó un total de 159 peces del género Achirus, siendo la especie más abundante Achirus klunzingeri (98), luego Achirus mazatlanus (44) y finalmente Achirus scutum (17). La dieta de A. scutum se basó en detritos, la de $A$. mazatlanus en detritos y peces y la de A. klunzingeri en crustáceos y peces. Los individuos de A. klunzingeri presentaron diferencias en su dieta entre la zona interna y externa del estuario, en la zona interna los peces fueron la presa principal y en la zona externa fueron los crustáceos. Las especies A. klunzingeri y A. mazatlanus presentaron ingesta de microplásticos, principalmente fibras alargadas e incoloras presentes en individuos de todas las tallas. La dinámica de los hábitos tróficos de $A$. klunzingeri, se relacionaron con algunas variables ambientales, como temperatura, trasparencia y oxígeno disuelto. Por ejemplo, estas tres variables presentaron correlaciones negativas con el ítem peces, y positivas para la categoría crustáceos.

Conclusión: Se evidenció que estas especies son generalistas y que las condiciones ambientales no solo influenciaron la dinámica del consumo alimentario, sino que también, determinaron la presencia de los microplásticos.

Palabras claves: Achirus; hábitos tróficos; fibras microplásticas; Pacífico Oriental Tropical; estuarios.

\section{REFERENCIAS}

Alessandro, M. D., Porporato, E. M. D., Esposito, V., Giacobbe, S., Deidun, A., Nasi, F., Ferrante, L., Auriemma, R., Berto, D., Renzi, M., Scotti, G., Consoli, P., del, P., Andaloro, F., \& Romeo, T. (2020). Common patterns of functional and biotic indices in response to multiple stressors in marine harbours ecosystems. Environmental Pollution, 259, 113959. https://doi. org/10.1016/j.envpol.2020.113959

Bárbara, V., \& Peña, I. (2006). Los fondos marinos de maërl del Parque Nacional de las Islas Atlánticas (Galicia, España): distribución, abundancia y flora asociada. Nova Acta Cientifica Compostelana (Bioloxía), 15, 7-25.

Bermúdez-guzmán, L., Alpízar-Villalobos, C., GatgensGarcía, J., Jiménez-Huezo, G., Rodríguez-Arias, M., Molina, H., Villalobos, J., Paniagua, S., Vega-Baudrit, J. R., \& Rojas-Jimenez, K. (2020). Microplastic ingestion by a herring Opisthonema sp. in the Pacific coast of Costa Rica. Regional Studies in Marine Science, 38, 101367. https://doi.org/10.1016/j. rsma.2020.101367

Cantera, J. R., \& Blanco, J. F. (2001). The Estuary Ecosystem of Buenaventura Bay, Colombia. In U. Seeliger, \& B. Kjerfve (Eds.), Coastal Marine Ecosystems of Latin America (pp. 265-280). Springer Berlin Heidelberg. https://doi.org/10.1007/978-3-662-04482-7_19

Cervera, L., Román, J., Osorio, N., Vázquez, C., Jesús, R. de, \& Densa, E. (2006). Crecimiento, producción y eficiencias de energía de crías de Acocil Cambarellus Montezumae (Saussure) alimentadas con detritus de Egeria densa. REDVET. Revista Electrónica de Veterinaria, $\operatorname{VII}(12), 1-11$.

Chango, A., \& Nacimba, N. (2015). Propuesta de un plan de monitoreo de estuarios y evaluación de calidad del agua. Caso de estudio: Estuario de la subcuenca del río Atacames. Escuela Politécnica Nacional, Quito, Ecuador. http://bibdigital.epn.edu. ec/handle/15000/14485

Clarke, K. R., Gorley, R. N., Somerfield, P. J., \& Warwick, R. M. (2014). Change in Marine Communities: An Approach to Statistical Analysis and Interpretation 3rd edition (3rd ed.). PRIMER-E: Plymouth.

Cogua, P., Jiménez, M. F., \& Duque, G. (2013). Relaciones tróficas de cinco especies de peces de interés comercial en la Bahía de Cartagena, Caribe Colombiano. Boletin de Investigaciones Marinas y Costeras, 42(1125), 185-192. 
Day, J., Yáñez, A., Kemp, W. M., \& Crump, B. C. (2012). Estuarine Ecology, 2, 54. https://doi org/10.1002/9781118412787.ch1

Dehaut, A., Hermabessiere, L., \& Duflos, G. (2019). Current frontiers and recommendations for the study of microplastics in seafood. TrAC - Trends in Analytical Chemistry, 116, 346-359. https://doi. org/10.1016/j.trac.2018.11.011

Du, Y., Dong, X., Jiang, X., Zhang, Y., Zhu, D., Sun, Q., Wang, Z., Niu, X., Chen, W., Zhu, C., Jing, Z., Tang, S., Li, Y., Chen, J., Chu, X., Xu, C., Wang, T., He, Y., Han, B., ... Peng, S. (2021). Ocean surface current multiscale observation mission (OSCOM): Simultaneous measurement of ocean surface current, vector wind, and temperature. Progress in Oceanography, 193. https://doi.org/10.1016/j.pocean.2021.102531

Duque, G., \& Acero, A. (2003a). Feeding Selectivity of Anchovia clupeoides (Pisces: Engraulidae) in the Ciénaga Grande de Santa Marta, Colombian Caribbean. Gulf and Caribbean Research, 15(1), 21-26. https://doi.org/10.18785/gcr.1501.04

Duque, G., \& Acero, A. (2003b). Foods habits of Anchovia clupeoides (Pisces: Engraulidae) in the Ciénaga Grande de Santa Marta, Colombian Caribbean. Gulf of Mexico Science, 21(1), 1-9. https://doi. org/10.18785/goms.2101.01

Duque, G., \& Acero, A. (2006). Ración diaria y consumo de alimento de la población de Anchovia Clupeoides (Pisces: Engraulidae) en la Ciénaga Grande de Santa Marta, Caribe colombiano. Boletín de Investigaciones Marinas y Costeras, 35(963), 207-221.

Duque, G., \& Cogua, P. (2016). Mercurio en peces de la bahía de Buenaventura. Ingenium, 10(29), 11-17. https:// repository.usc.edu.co/bitstream/20.500.12421/794/1/ MERCUR 1.PDF

Duque, G., Gamboa-García, D. E., Molina, A., \& Cogua, P. (2020). Effect of water quality variation on fish assemblages in an anthropogenically impacted tropical estuary, Colombian Pacific. https://doi. org/10.1007/s11356-020-08971-2

Elías, R. (2015). Mar del plástico: una revisión del plástico en el mar. Revista de Investigación y Desarrollo Pesquero, 105, 83-105.

Enriquez, O., Guzmán, A., \& Narváez, G. (2014). Analysis of Precipitation Behavior in the Municipality of Buenaventura (Valle del Cauca, Colombia) in the Context of the El Niño/La Niña Phenomenon. Revista Colombiana de Geografia, 23(1), 65-178.

Esposito, V., Castriota, L., Consoli, P., Romeo, T., Falautano, M., \& Andaloro, F. (2010). Feeding habits and selectivity of the wide-eyed flounder, Bothus podas (Delaroche, 1809) (Bothidae) from the southern Tyrrhenian sea. Marine Biology Research, 6, 496-502. https://doi.org/10.1080/17451000903438495
Fischer, W., Krupp, F., Schneider, W., Sommer, C., Carpenter, K. E., \& Niem, V. H. (1995). Guía FAO para la identificación de especies para los fines de la pesca. Pacífico centro-oriental. Volumen II. Vertebrados Parte 1. FAO.

Flores, J., Godínez, E., Gaspar, S., Rojo, J., López, A., \& Morales, M. (2013). Abundancia, distribución, hábitos alimentarios e interacciones tróficas de cinco especies de lenguados (Pleuronectiformes) en el Pacífico central mexicano. Latin American Journal of Aquatic Research, 41(3), 423-439. https://doi. org/103856/vol41-issue3-fulltext-6

Flores, J. R., Godínez, E., \& Gaspar, S. (2015). Ecología trófica de siete especies de batoideos (Batoidea) en el Pacífico Central Mexicano. Revista de Biología Marina y Oceanografia, 50(9), 521-533. https://doi. org/10.1017/CBO9781107415324.004

França, S., Costa, M. J., \& Cabral, H. N. (2011). Interand intra-estuarine fish assemblage variability patterns along the Portuguese coast. Estuarine, Coastal and Shelf Science, 91(2), 262-271. https://doi. org/10.1016/j.ecss.2010.10.035

Froese, R., \& Pauly, D. (2019). Fishbase. World Wide Web electronic publication. http://fishbase.org/search.php

Gallego Perez, B. E., \& Selvaraj, J. J. (2019). Evaluation of coastal vulnerability for the District of Buenaventura, Colombia: A geospatial approach. Remote Sensing Applications: Society and Environment, 16(April), 100263. https://doi.org/10.1016/j.rsase.2019.100263

Galvis, O., Cueto, A., Navarro, Y., \& González, P. (2005). Consumo de oxígeno de juveniles de tilapia (Oreochromis niloticus) bajo diferentes condiciones de temperatura y su relación con la alimentación. Revista Dugandia, Ciencias Básicas, Uniatlántico, 1(1), 102-110. https://pdfs.semanticscholar.org/4877/fcebe339be0f2cb73e7caef2d9e7af751175.pdf

Gamboa-García, D., Duque, G., \& Cogua, P. (2018). Structural and compositional dynamics of macroinvertebrates and their relation to environmental variables in Buenaventura Bay. Boletin de Investigaciones Marinas y Costeras, 47(1), 67-83. https://doi. org/10.25268/bimc.invemar.2018.47.1.738

Gonzalo, T. (2011). Ecología trófica del lenguado Paralichthys patagonicus (Jordan, 1889) en el Ecosistema Costero Argentino-Uruguayo (Tesis Licenciatura). Universidad Nacional de Mar Del Plata, Argentina.

Grabowska, J., Kakareko, T., Błońska, D., Przybylski, M., Kobak, J., Jermacz, Ł., \& Copp, G. H. (2016). Interspecific competition for a shelter between nonnative racer goby and native European bullhead under experimental conditions - Effects of season, fish size and light conditions. Limnologica, 56, 30-38. https:// doi.org/10.1016/J.LIMNO.2015.11.004 
Guevara, E., Álvarez, H., Mascaró, M., Rosas, C., \& Sánchez, A. (2007). Hábitos alimenticios y ecología trófica del pez Lutjanus griseus (Pisces: Lutjanidae) asociado a la vegetación sumergida en la Laguna de Términos, Campeche, México. Revista de Biología Tropical, 55(3-4), 989-1004.

Guo, X., \& Wang, J. (2019). The chemical behaviors of microplastics in marine environment: A review. Marine Pollution Bulletin, 142, 1-14. https://doi. org/10.1016/j.marpolbul.2019.03.019

López, R., \& Arcila, C. (2002). Diet Composition of Fish Species from the Southern Continental Shelf of Colombia. Naga, WorldFish Center Quarterly, 25(34), 23-29.

Lusher, A. L., McHugh, M., \& Thompson, R. C. (2013). Occurrence of microplastics in the gastrointestinal tract of pelagic and demersal fish from the English Channel. Marine Pollution Bulletin, 67(1-2), 94-99. https://doi.org/10.1016/j.marpolbul.2012.11.028

Lusher, A. L., Welden, N. A., Sobral, P., \& Cole, M. (2017). Sampling, isolating and identifying microplastics ingested by fish and invertebrates. Analytical Methods, 9(9), 119-148. https://doi.org/10.1039/ c6ay02415g

Majdi, N., Hette, N., Auclair, E., Bec, A., Chouvelon, T., Cognie, B., Danger, M., Decottignies, P., Dessier, A., Desvilettes, C., Dubois, S., Dupuy, C., Fritsch, C., Gaucherel, C., Hedde, M., Jabot, F., Lefebvre, S., Marzloff, M. P., Pey, B., ... Perga, M. (2018). There's no harm in having too much: A comprehensive toolbox of methods in trophic ecology. Food Webs, 16, e00100. https://doi.org/10.1016/j.fooweb.2018. $\mathrm{e} 00100$

Marques, J. F., Teixeira, C., Pinheiro, A., Peschke, K., \& Cabral, H. (2009). A multivariate approach to the feeding ecology of the Channel flounder, Syacium micrurum (Pisces, Pleuronectiformes), in Cape Verde, Eastern Atlantic. Ciencias Marinas, 35, 15-27.

Marti, A. (2017). Permutational Multivariate Analysis of Variance (PERMANOVA). Wiley statsref: statistics reference online, 1-15. https://doi. org/10.1002/9781118445112.stat07841

Martin, J., Lusher, A., Thompson, R. C., \& Morley, A. (2017). The Deposition and Accumulation of Microplastics in Marine Sediments and Bottom Water from the Irish Continental Shelf. Scientific Reports, 7(1), 1-9. https://doi.org/10.1038/s41598-017-11079-2

Martinez, O., Molina, A. E., \& Duque, G. (2019). Influencia de la materia orgánica en la abundancia de poliquetos (Annelida: Polychaeta) de la bahía de Buenaventura, Pacífico colombiano. In N. H. Campos \& A. Acero (Eds.), Ciencias del mar - Una mirada desde la Universidad Nacional de Colombia ( $1^{\text {st }}$ Ed., pp. 228). Editorial Universidad Nacional de Colombia.
Martinez, R., Tuyas, L., Martinez, M., Pérez, A., \& Cánovas, A. (2009). El coeficiente de correlación de los rangos de Spearman caracterización. Revista Habanera de Ciencias Médicas, 8(2), 1-19.

McGoran, A. R., Clark, P. F., \& Morritt, D. (2017). Presence of microplastic in the digestive tracts of European flounder, Platichthys flesus, and European smelt, Osmerus eperlanus, from the River Thames. Environmental Pollution, 220, 744-751. https://doi. org/10.1016/j.envpol.2016.09.078

Molina, A., Duque, G., \& Cogua, P. (2020). Influences of environmental conditions in the fish assemblage structure of a tropical estuary. Marine Biodiversity, $50(1), 1-13$.

Mu, J., Qu, L., Jin, F., Zhang, S., Fang, C., \& Ma, X. (2019). Abundance and distribution of microplastics in the surface sediments from the northern Bering and Chukchi Seas. Environmental Pollution, 245, 122130. https://doi.org/10.1016/j.envpol.2018.10.097

Munday, P., Jones, G., \& Caley, J. (2001). Interspecific competition and coexistence in a guild of coraldwelling fishes. Ecology, 82(8), 2177-2189.

Nelson, J. (2006). Fishes of the World (4 $4^{\text {th }}$ Ed.). John Wiley \& Sons.

Norbis, W., \& Galli, O. (2004). Hábitos de alimentación del lenguado Paralichtys orbignyanus (Valenciennes, 1842) en una laguna costera somera del Atlántico Sur: Rocha , Uruguay. Ciencias Marinas, 30(4), 619-626.

Olaya, C., \& Arellano, J. (2012). Feeding Habits of Barbul de Piedra (Ariopsis sp.) in the Sinu River, Colombia. Acta Biológica Colombiana, 17(1), 117-128.

Ory, N., Chagnon, C., Felix, F., Fernández, C., Lia, J., Gallardo, C., Garcés, O., Henostroza, A., Laaz, E., Mizraji, R., Mojica, H., Murillo, V., Ossa, L., Preciado, M., Sobral, P., Urbina, M. A., \& Thiel, M. (2018). Low prevalence of microplastic contamination in planktivorous fish species from the southeast Pacific Ocean. Marine Pollution Bulletin, 127, 211-216. https://doi.org/10.1016/j.marpolbul.2017.12.016

Otero, E., Mosquera, L., Silva, G., \& Guzmán, J. (2007). Deltas y Estuarios de Colombia. Colección Ecológica del Banco de Occidente. Banco de Occidente. https://www.imeditores.com/banocc/deltas/cap7.htm

Otero, L. (2005). Aplicación de un modelo hidrodinámico bidimensional para describir las corrientes y la propagación de la onda de marea en la bahía de Buenaventura. Boletín Científico CCCP, 12, 9-21. https://doi. org/10.26640/01213423.12.9

Peña, J., \& Palacios, M. (2013). Biodiversity as a Strategy for Sustainable Development in the Colombian Pacific: Some approaches to its Management. Revista del Doctorado Interinstitucional en Ciencias Ambientales, 3, 37-43. 
Peng, G., Zhu, B., Yang, D., Su, L., Shi, H., \& Li, D. (2017). Microplastics in sediments of the Changjiang Estuary, China. Environmental Pollution, 225, 283290. https://doi.org/10.1016/j.envpol.2016.12.064

Pérez, G. (2007). Historia, geografia y puerto como determinantes de la situación social de buenaventura. Documentos de Trabajo Sobre Economía Regional (Vol. 91). Banco de la República Colombia.

Pichler, H. A., Spach, H. L., Gray, C. A., Broadhurst, M K., Schwarz, R., \& de Oliveira Neto, J. F. (2015). Environmental influences on resident and transient fishes across shallow estuarine beaches and tidal flats in a Brazilian World Heritage area. Estuarine, Coastal and Shelf Science, 164, 482-492. https://doi. org/10.1016/j.ecss.2015.07.041

Pozzobon, M. V. (1987). Estudios preliminares sobre las interrelaciones tróficas de 20 especies de peces del área costera bonaerense (Tesis de licenciatura). Universidad Nacional de Mar del Plata, Argentina. https://www.oceandocs.org/handle/1834/3555

Riascos, J. M., Valencia, N., Peña, E. J., \& Cantera, J. R. (2019). Inhabiting the technosphere: The encroachment of anthropogenic marine litter in Neotropical mangrove forests and its use as habitat by macrobenthic biota. Marine Pollution Bulletin, 142, 559568. https://doi.org/10.1016/j.marpolbul.2019.04.010

Robertson, D., \& Allen, G. (2015). Shorefishes of the Tropical Eastern Pacific: Online information system. Smithsonian Tropical Research Institute. https://biogeodb.stri.si.edu/sftep/es/pages

Rodríguez, J., \& Reul, A. (2010). Biodiversidad y servicios de los ecosistemas marinos. Uciencia: revista de divulgación científica de la Universidad de Málaga. https://core.ac.uk/download/pdf/62896243.pdf
Sandoval, E., Madrigal, X., Escalera, L., Medina, M., \& Domínguez, O. (2014). Estructura de la comunidad de peces en cuatro estuarios del Pacífico mexicano central. Revista Mexicana de Biodiversidad, 85(4), 1184-1196. https://doi.org/10.7550/rmb.42105

Sosa-López, A., Mouillot, D., Ramos-Miranda, J., FloresHernandez, D., \& Chi, T. Do. (2007). Fish species richness decreases with salinity in tropical coastal lagoons. Journal of Biogeography, 34(1), 52-61. https://doi.org/10.1111/J.1365-2699.2006.01588.X

Sousa, L., Silva, B., Nascimieno, M., \& Machado, A. (2019). Variacao sazonal das pastículas de microplásticos no sedimento de quatro praiass urbanas no estado da Paraiba, Brasil. IV Congresso Nacional de Pesquisa e Ensino em Ciências CONAPESC, Campina Grande, Brasil, Agosto 2019.

Vásquez, D., Molina, A., \& Duque, G. (2021). Distribución espacial y aumento a través del tiempo de microplásticos en sedimentos de la Bahía de Buenaventura, Pacífico colombiano. Boletín de Investigaciones Marinas y Costeras, 50(1), 27-42.

Wright, S. L., Thompson, R. C., \& Galloway, T. S. (2013). The physical impacts of microplastics on marine organisms: a review. Environmental Pollution, 178, 483-492.

Zhang, C., Zhou, H., Cui, Y., Wang, C., Li, Y., \& Zhang, D. (2019). Microplastics in offshore sediment in the Yellow Sea and East China Sea, China. Environmental Pollution, 244, 827-833. https://doi.org/10.1016/j. envpol.2018.10.102

Zhang, D., Liu, X., Huang, W., Li, J., Wang, C., Zhang, D., \& Zhang, C. (2020). Microplastic pollution in deepsea sediments and organisms of the Western Pacific Ocean. Environmental Pollution, 259, 113948. https://doi.org/10.1016/j.envpol.2020.113948 